\section{Kommentar}

Der primäre Hyperaldosteronismus ist eine der häufigsten sekundären Hypertonieursachen. Auch bei langjährig bestehender arterieller Hypertonie muss an einen primären Hyperaldosteronismus gedacht werden. Bei diesem Patienten war die Hypokaliämie bereits sehr verdächtig. Man muss jedoch wissen, dass ein Großteil der Patienten mit primärem Hyperaldosteronismus normokaliämisch sind. Die häufigste Ursache des primären Hyperaldosteronismus sind die bilaterale Hyperplasie oder einseitige Adenome. Die Abklärung erfolgt nach einem standardisierten Schema. Als Screening ist der Aldosteron/Renin-Quotient (ARQ) am besten geeignet. Im Idealfall sind die Antihypertensiva, insbesondere Betablocker, vorher abzusetzen (1 Woche) und in jedem Fall die Aldosteronantagonisten und kaliumsparende Diuretika (4 Wochen). Bei erhöhtem ARQ ist ein Bestätigungstest zwingend notwendig, den wir als Kochsalzbelastungstest durchführen.
Hierdurch wird die Autonomie der Aldosteronproduktion nachgewiesen. Normalerweise fällt Aldosteron unter $50 \mathrm{pg} / \mathrm{ml}$, Werte zwischen $50-100 \mathrm{pg} / \mathrm{ml}$ stellen einen Graubereich dar. Bei Werten über 100 $\mathrm{pg} / \mathrm{ml}$ nach Kochsalzbelastung wie in diesem Fall ist die Diagnose gesichert.

Da der Patient der Operation eines evtl. vorliegenden Adenoms aufgeschlossen gegenüberstand, wurde eine weitere Differenzialdiagnose mit Bildgebung (CT ist ausreichend) und seitengetrennter Nebennierenvenenblutentnahme durchgeführt, die eine ausgeprägte Lateralisierung der Aldosteronproduktion zeigte. Die Untersuchung ist technisch nicht einfach und nur spezialisierten Zentren vorbehalten. Eine selektive Katheterisierung (Cortisol in der Nebennierenvene im Vergleich zu Vena cava) ist für die Auswertung unbeding notwendig.

Ein negatives CT schließt bei biochemisch nachgewiesenen primären Hyperaldosteronismus Mikroadenome nicht aus. Der operative Goldstandard ist die retroperitoneoskopische Adrenalektomie. Wir empfehlen eine Vorbehandlung mit einem Mineralokortikoid-Rezeptor-Antagonisten z.B. Spironolakton $50-100 \mathrm{mg} / \mathrm{d}$ für 2-4 Wochen vor Operation. Auf das Absetzen von Spironolakton nach der Operation ist unbedingt zu achten. Prinzipiell ist bei Nachweis eines Adenoms und/oder klarer Lateralisierung des AC-Quotienten (> 3) die Operation vorzuziehen, da Mineralokortikoid-Rezeptor-Antagonisten nicht alle negativen Effekte des Aldosterons blockieren.

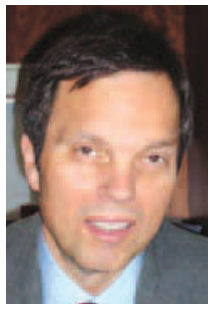

Korrespondenzanschrift: Prof. Dr. L. C. Rump Karen Eschweiler Klinik für Nephrologie Universitätsklinikum Düsseldorf Moorenstraße 5 D-40225 Düsseldorf E-Mail:Christian.Rump@med. uni-duesseldorf.de \title{
Themenwochen „Starkes Herz": Bringen Sie mehr Herz ins Patientengespräch
}

SBK setzt auf die Zusammenarbeit mit Haus- und Fachärzten

I Ein halbes Jahr lang steht bei der SBK (Siemens-Betriebskrankenkasse) die Herzgesundheit ihrer Versicherten ganz oben auf der Tagesordnung. Mit den Themenwochen „Starkes Herz“, die am 18. Oktober 2010 gestartet sind, möchte die Kasse ihre Versicherten zu einem herzgesunden Lebensstil motivieren. Bis Mitte März 2011 finden zahlreiche Aktionen und Veranstaltungen statt, bei denen alltagstaugliche Tipps rund um die Themen Bewegung, Ernährung oder Stressabbau gegeben werden. Die Botschaft: Man kann selbst viel tun, damit das Herz gesund bleibt.

Die Themenwochen „Starkes Herz“ will die SBK auch dazu nutzen, um für den KardioPro Risikocheck zu werben. Daran können alle Versicherten zwischen 45 und 75 Jahren ohne Koronarerkrankung teilnehmen, die ihr Herzinfarktrisiko in Erfahrung bringen möchten. Dieser Risikocheck basiert auf dem PROCAM-Gesund- heitstest und wird beim Hausarzt durchgeführt. Es wird dabei das individuelle Risiko in den nächsten zehn Jahren einen Herzinfarkt zu erleiden, bestimmt. Wenn die Untersuchung ein mittleres oder hohes kardiovaskuläres Risiko ergibt, sind weitere diagnostische Schritte innerhalb des regional angebotenen Vorsorge- und Versorgungsprogramms KardioPro bei einem kardiologischen Vertragspartner der SBK vorgesehen. Für interessierte Praxen und Kliniken stellt die SBK deshalb begleitend zu den Themenwochen eine Fülle von Informationsmaterialien zur Verfügung, die im Wartezimmer ausgelegt werden können oder die der Arzt im Beratungsgespräch nutzen kann. So hält die SBK beispielsweise eine Reihe von sogenannten ImpulseRatgeberkarten mit Tipps zur gesunden Ernährung oder zum Stressabbau bereit.

Holen Sie sich die SBK Themenwochen „Starkes Herz“ in Ihre Praxis und

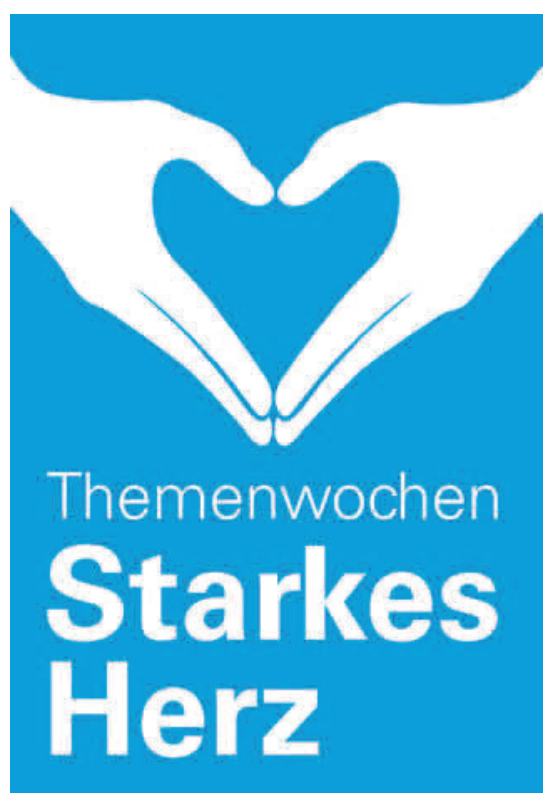

fordern Sie jetzt die begleitenden Unterlagen an:

SBK Zentrale

Frau Susanne Weber

Tel.: 07321 303-143

E-Mail: susanne.weber@sbk.org 